\title{
High coupling efficiency to a low dispersion slow light-supporting photonic crystal waveguide
}

\author{
M. Khatibi Moghaddam \\ ma_kh303@stu-mail.um.ac.ir
}

\section{A. R. Attari}

\section{M. Mirsalehi}

\begin{abstract}
Communications and Computer Research Center, Ferdowsi University of Mashhad, Iran
Department of Electrical Engineering, Ferdowsi University of Mashhad, Iran

Department of Electrical Engineering, Ferdowsi University of Mashhad, Iran
\end{abstract}

Department of Electrical Engineering, Ferdowsi University of Mashhad, Iran

In this paper, we design a waveguide on photonic crystal slab for propagation of low-dispersion slow light. By shifting the air holes adjacent to the waveguide, we obtain a photonic crystal waveguide with a group index of 25 in $25 \mathrm{~nm}$ bandwidth which results in a group index bandwidth product of 0.366 . To take the advantages of low dispersion slow light generated in this engineered waveguide, we next focus on low coupling efficiency limitation. A low group index coupler is proposed to increase the transmission of the light to the slow mode in the low dispersion bandwidth. By using the proposed coupler and adjusting the structural parameters, the coupling efficiency to low dispersion slow light is improved $11 \mathrm{~dB}$ compared to the transmission without the coupler.

[DOI: http://dx.doi.org/10.2971/jeos.2013.13066]

Keywords: Slow light, photonic crystal, group velocity dispersion, coupling

\section{INTRODUCTION}

Slow light technology plays an important role in the future of optical buffers, optical logical gates, and optical signal processing. Photonic crystal waveguide (PCW) is widely used in generation of slow light. In a PCW slow light can be realized by modifying its structure. This works for a desired wavelength and also provides larger bandwidths compared with other methods, such as electromagnetically induced transparency. The slow light generated in a PCW can be used in several applications, such as optical delay lines or buffers and enhanced light-matter interaction [1]-[3].

There are two main limitations in realization of slow light in PCW: 1) the group velocity dispersion (GVD) in slow light region, and 2) low efficiency of coupling light from strip waveguide to the slow light PCW. GVD occurs when the group velocity of light depends on optical frequency or wavelength. The GVD parameter is defined as $\beta_{2}=\partial / \partial \omega\left(1 / v_{g}\right)=\left(\partial^{2} k\right) /\left(\partial \omega^{2}\right)$, where $k$ is the phase constant, $\omega$ is the angular frequency and $v_{g}$ is the group velocity of signal. In optical communications, the dispersion parameter $D_{\lambda}$ is defined as

$$
D_{\lambda}=\frac{\mathrm{d}}{\mathrm{d} \lambda}\left(\frac{1}{v_{g}}\right)=\frac{-2 \pi c}{\lambda^{2}} \beta_{2}
$$

where $\lambda$ is the optical wavelength and $c$ is the velocity of light in free space. High group index $\left(n_{g}=c / v_{g}\right)$ is usually achieved in the band edge of a conventional PCW. It has a narrow bandwidth associated with a high GVD [2]-[4] which results in pulse broadening. These limitations can be overcome by engineering the dispersion relation of the PCW guided mode [4]. Several methods are used to decrease GVD and obtain high group index in wide bandwidth [5]-[14]. The main method is waveguide engineering to obtain a PCW with nearly zero dispersion in an acceptable bandwidth.

Since the bandwidth of slow light becomes narrower as $n_{g}$ increases, we define group index-bandwidth product (GBP) according to Eq. (2),

$$
\mathrm{GBP}=\left(\frac{\Delta \omega}{\omega_{0}} \tilde{n}_{g}\right)
$$

as a figure of merit for waveguides, where $\tilde{n}_{g}$ is the average group index, $\omega_{0}$ is the central angular frequency, and the bandwidth $\Delta \omega$ is defined as the angular frequency range in which the variation of $n_{g}$ with respect to its mean value is less than $10 \%$ [9]-[11], [14].

The knowledge of field distribution in a PCW can be exploited to control dispersion properties of the guided modes that support the slow light. A PCW guided mode is formed by interaction of "index-guided" and "gap-guided" modes. This interaction determines the shape of dispersion curve and it can be altered by changing the index-guided mode, e.g., by changing the width of the line defect $[6,7]$. Also, changing the gap-guided mode which is obtained by changing the structure of the lattice is used in many dispersion engineering methods [8]-[14]. Dispersion engineered slow light PCW can be used in an optical switch or modulator to obtain a greater phase shifts in smaller switching lengths and lower modulation voltages $[15,16]$. In these structures which can be used in dense wavelength division demultiplexing (DWDM) systems, the optical bandwidth is an important parameter. This param- 
eter which is limited to the bandwidth of slow light should be more than $20 \mathrm{~nm}$ in DWDM. In PCW Mach-Zehnder modulator proposed in Ref. [15], the obtained bandwidth is about $10 \mathrm{~nm}$ due to the limited bandwidth of the waveguide used in this research. Recently, a slow light PCW has been proposed for construction of a photonic crystal optical modulator with a bandwidth of $18 \mathrm{~nm}$ [16].

Another limitation of slow light in PCW is the impedance mismatch between the strip waveguide and PCW modes in slow light regime which leads to low coupling efficiency [17]-[24]. Using a group index taper [20]-[22], and utilizing a fast light section as an interface between strip waveguide and PCW are the two main methods for increasing coupling efficiency [23, 24]. As shown in Ref. [24], the latter method results in higher transmission.

Couplers presented in previous papers were not designed for a low dispersion slow light in PCW and the signal pulse coupled in the waveguide suffered from dispersion. In this paper, we consider both dispersion and coupling limitations. Our goal is to obtain zero dispersion slow light in a PCW with a GBP higher than previously reported values and also to achieve high coupling efficiency from a strip waveguide to the designed slow light PCW. First, we improve the dispersion of the waveguide by modifying the structure of the lattice adjacent to the waveguide. Then, we evaluate coupling of a strip waveguide to the engineered PCW in low dispersion slow light bandwidth and propose a coupling PCW structure that supports low group index guided mode. We also modify the interface of the strip waveguide and the coupler to obtain higher transmission.

\section{DESIGN OF LOW DISPERSION SLOW LIGHT-SUPPORTING PCW}

The PCW considered in this paper is a W1 waveguide which is obtained by removing one row of air holes in a twodimensional triangular lattice of air holes of radius $r=0.286 a$, where $a$ is the PC lattice constant. The photonic crystal slab has silicon on insulator (SOI) structure composed of a silicon layer of $283 \mathrm{~nm}$ thickness on top and a silica layer of $2 \mathrm{~mm}$ thickness at the bottom [10]. Figure 1 shows the structure of PCW and design parameters. The even guided mode in W1 waveguide can be easily excited by a strip waveguide as shown in this figure.

We investigated the effects of several factors, such as PCW width $(d)$, radius of air holes in lattice near the waveguide, introducing air holes in the waveguide (with variable sizes and positions), and introducing an air slot in the waveguide. The results showed that changing the position of air holes near the waveguide affects the gap-guided mode more than other factors. Also, changing the position of air holes has less practical limitations. For example, reduction of waveguide width increases the scattering losses, since it increases the overlap of the optical mode with the holes.

In this paper, we modify the PCW structure by changing the position of air holes adjacent to the waveguide. This results in

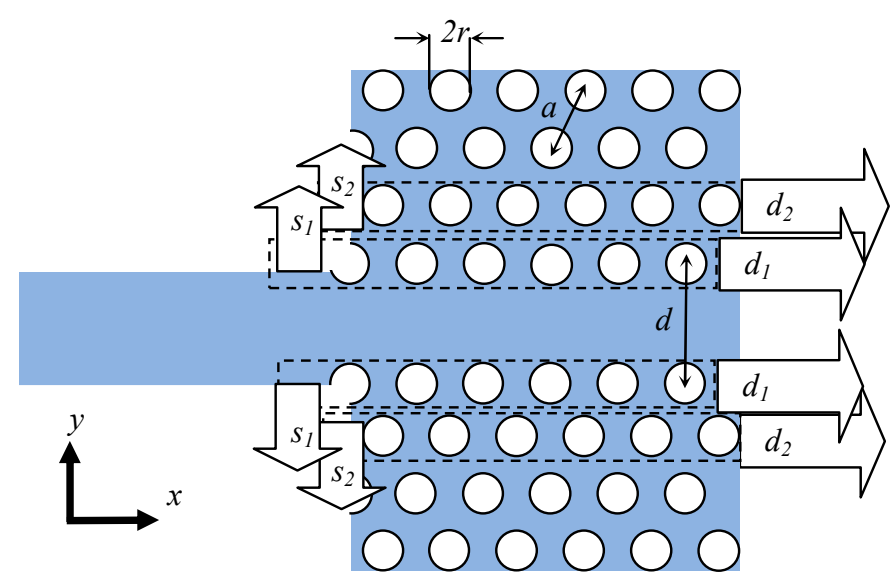

FIG. 1 PCW structure and design parameters.

changing the lattice structure surrounding the PCW and introduces guided modes which are different from W1 modes. The dispersion relation $\omega(k)$ of guided modes can be computed numerically using the Plane Wave Expansion (PWE) method by RSoft 2D Bandsolve. Due to the computational cost of three-dimensional simulations, we used two-dimensional simulations using slab effective index of 2.98 .

In PWE method, the numerical resolution of the lattice is an important parameter. Using a finer grid increases the accuracy of the calculation at the cost of longer computation time [25]. Therefore, it is logical to begin with a rough resolution and make it fine to obtain consistent results. Figure 2(a) shows the W1 guided mode for different number of plane waves $(N)$ in 3D PWE. To study the convergence of results by increasing $N$, Figure 2(b) shows the normalized cut off frequency of guided mode in band edge for different values of $N$. For $N>16$, the normalized cut off frequency difference reduces to $\Delta\left(a / \lambda_{c}\right)<0.0001$. Hence, we use $N=16$ in our PWE analyses.

Parameters that correspond to the air holes shifting are $d_{1}, d_{2}$, $s_{1}$ and $s_{2}$ as shown in Figure 1. These are the shifts of air holes in the first and second rows adjacent to the waveguide, parallel ( $x$ direction) and perpendicular ( $y$ direction) to the propagation direction. Shifting the first adjacent rows of air holes affects both the index-guided mode, whose profile is concentrated mostly in the waveguide, and gap-guided mode, whose profile has overlaps with three or four rows near the waveguide. Figure 3(a) shows that shifting the first row parallel to the waveguide more than $0.2 a\left(d_{1}>0.2 a\right)$ results in a minimum frequency for a wave number less than $0.4[2 \pi / a]$ in the dispersion curves. In these cases, we can obtain flat band slow light for wave numbers of $0.37-0.5[2 \pi / a]$. In this range, although the average group index reduces for high values of $d_{1}$, the GBP increases because of large bandwidth.

Perpendicular shift of the first adjacent row $\left(s_{1}\right)$ also alters the dispersion properties and produces guided modes which can support slow waves. It in fact changes the waveguide width and affects the index-guided modes. Figure 3(b) shows the dispersion curve of even guided mode for different values of $s_{1}$ while $d_{1}$ is kept constant at $0.5 a$. As shown in Figure 3(b), in some cases, such as $s_{1}=0.1 a$, a double mode frequency 


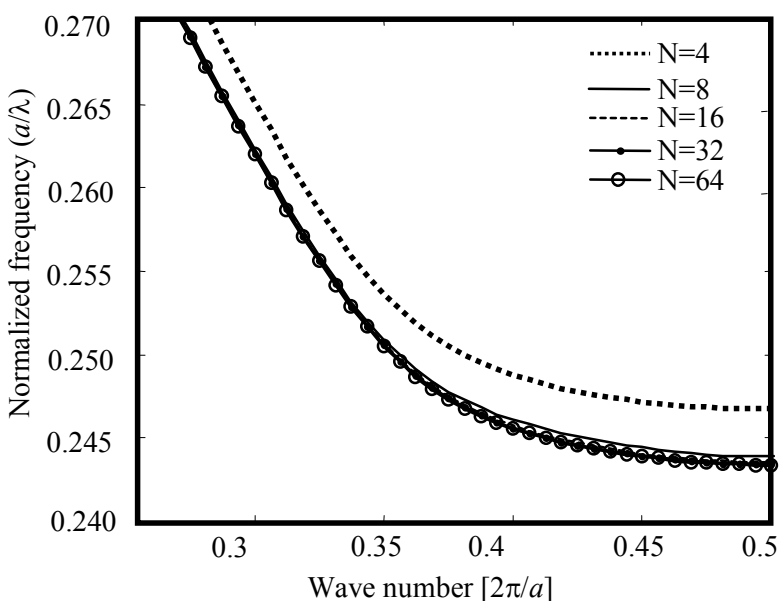

(a)

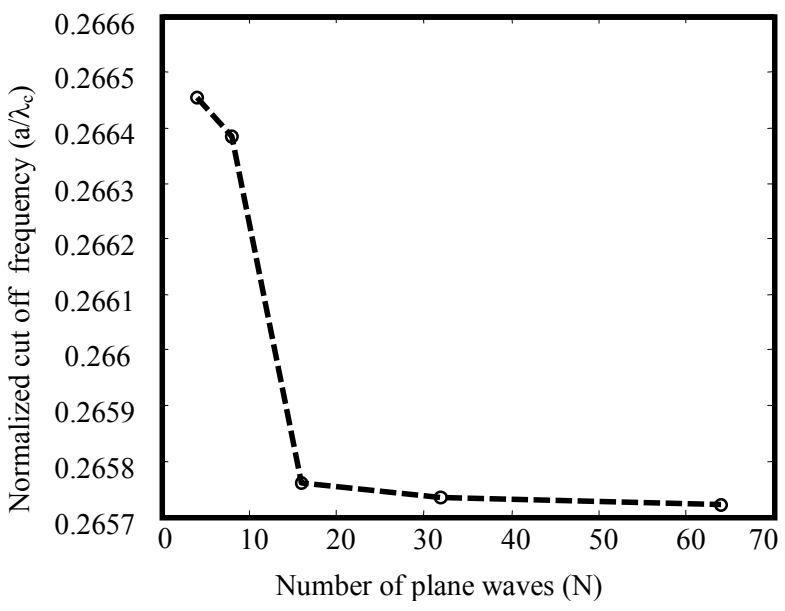

(b)

FIG. 2 (a) W1 guided mode for different values of $N$, (b) Variation of normalized cut off frequency of guided mode versus the number of plane waves.

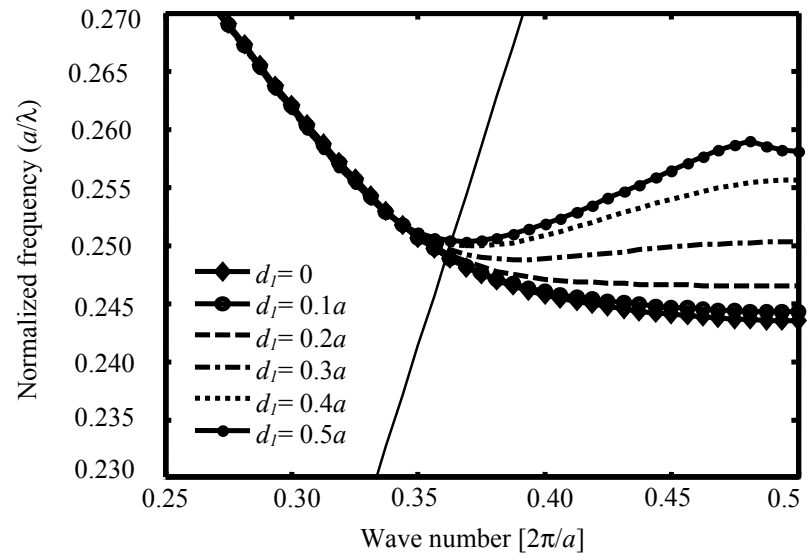

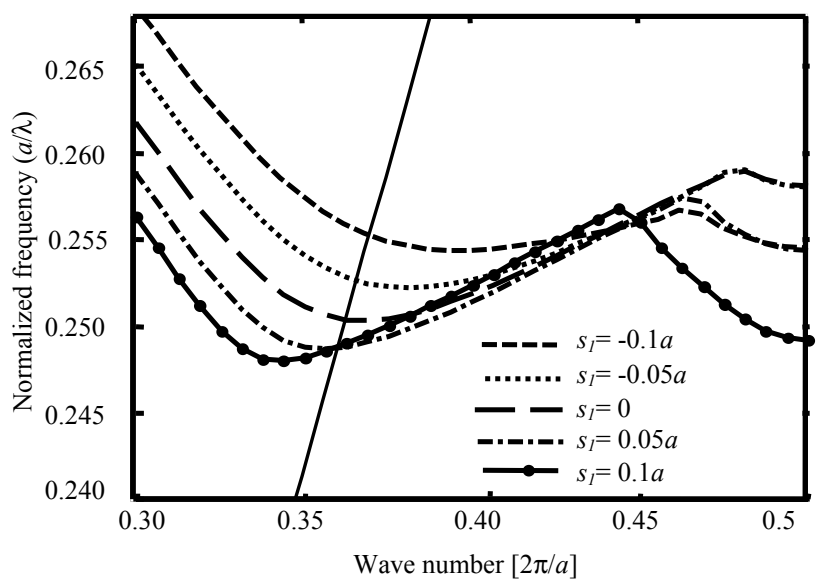

(b)

FIG. 3 (a) Dispersion curve of the even guided mode for different values of $d_{1}$. (b) Dispersion curve of the even guided mode for different values of $s_{1}\left(d_{1}=0.5 a\right)$. The straight line is the light line.

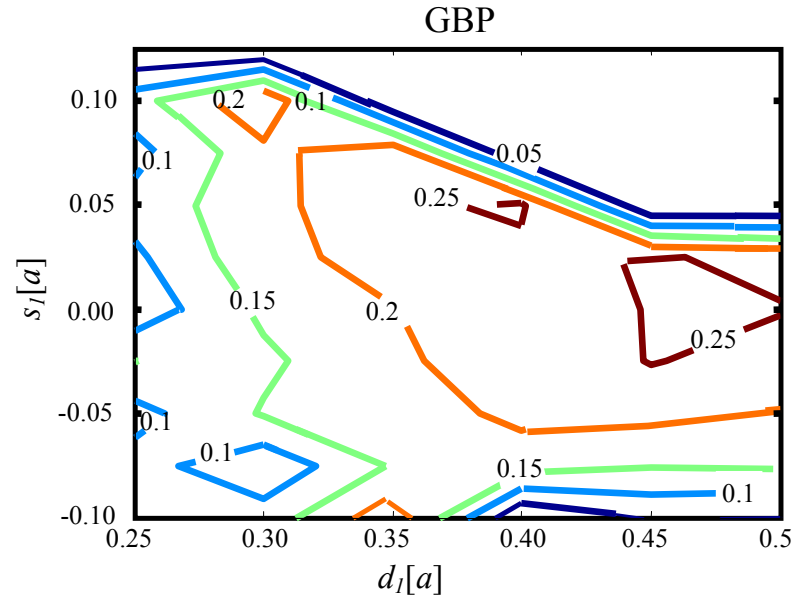

(a)

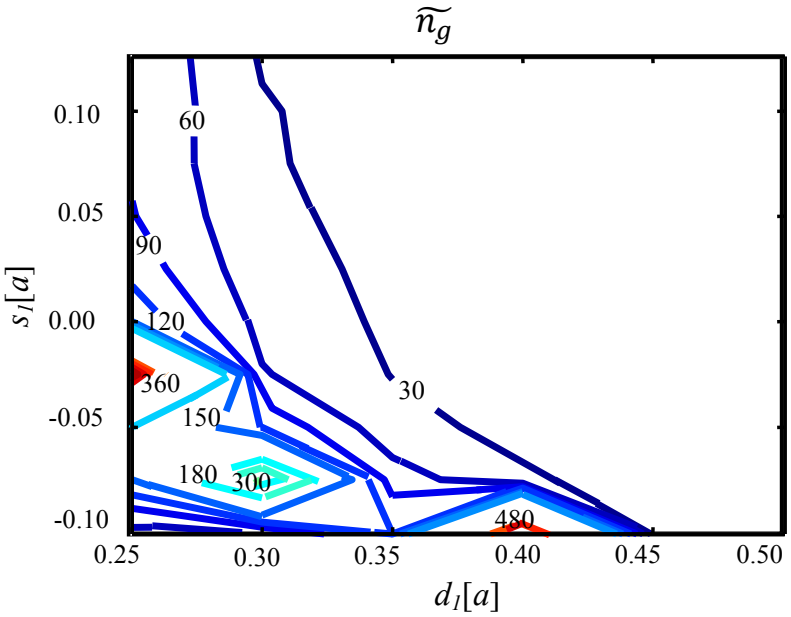

(b)

FIG. 4 (a) GBP and (b) average group index versus $d_{1}$ and $s_{1}$.

band $(0.249-0.257(a / \lambda))$ appears. Although there are zero dispersion regions in these cases, they are not single mode and hence are not useful. The bandwidths of double mode regions are not included in Eq. (2) for GBP calculation.

In the first step, we investigate the effects of parallel and perpendicular shifts of the first adjacent row $\left(d_{1}, s_{1}\right)$ simultaneously. We find that zero dispersion slow light can be obtained for $0.25 a<d_{1}<0.5 a$ and $-0.1 a<s_{1}<0.1 a$. Figures 4(a) and 4 (b) respectively show the GBP and average group index for various values of $d_{1}$ and $s_{1}$. There are some cases with GBP values of $0.25-0.26$, but their corresponding average group indices $\left(\tilde{n}_{g}\right)$ are less than 14 . The best case (maximum GBP) that satisfies the $\tilde{n}_{g}>20$ constraint is a structure with $d_{1}=0.3 a$ and $s_{1}=0.1 a$. The corresponding value of this case 


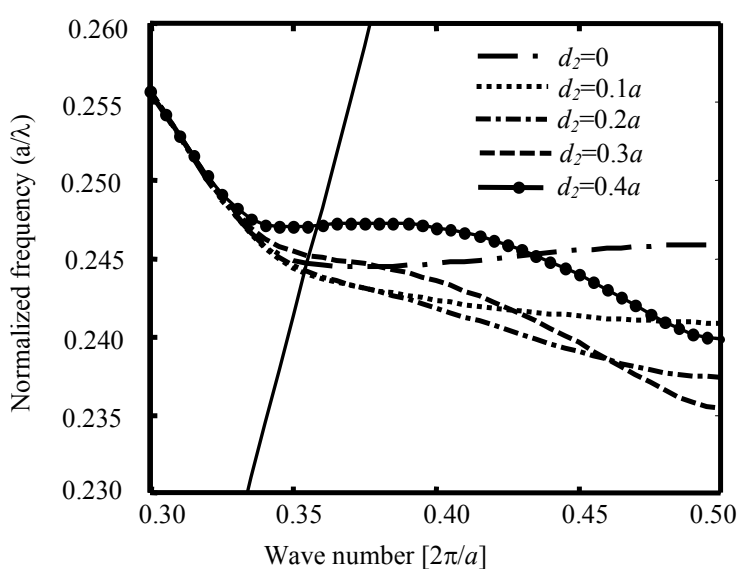

(a)

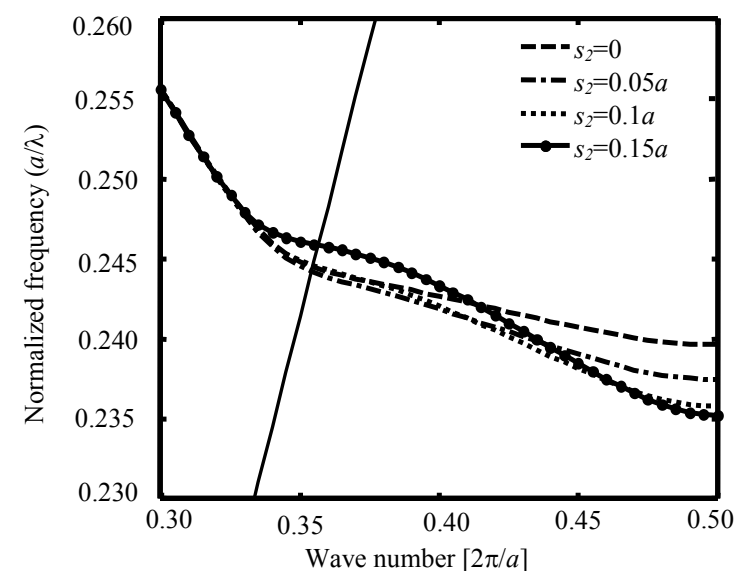

(b)

FIG. 5 (a) Dispersion curve of the even guided mode for different values of $d_{2}\left(s_{2}=0.05 a\right)$. (b) Dispersion curve of even guided mode for different values of $s_{2}\left(d_{2}=0.2 a\right.$ ). The straight line is the light line.

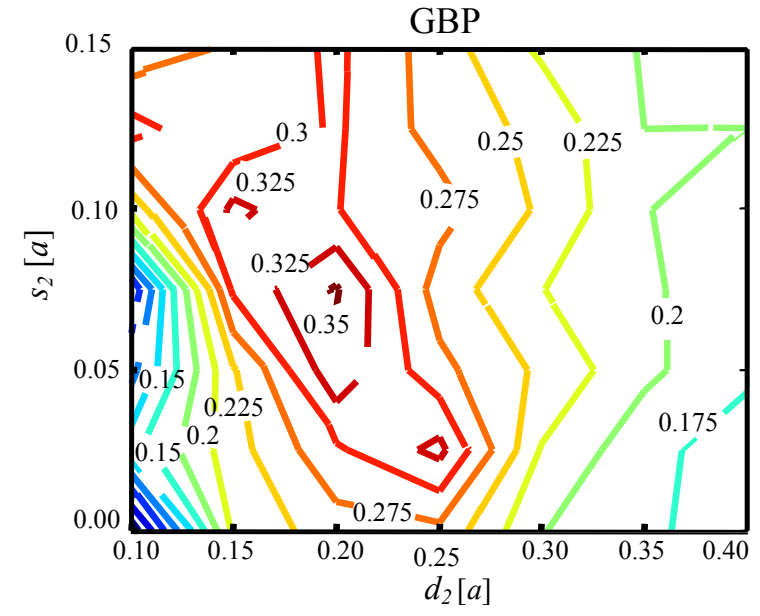

(a)

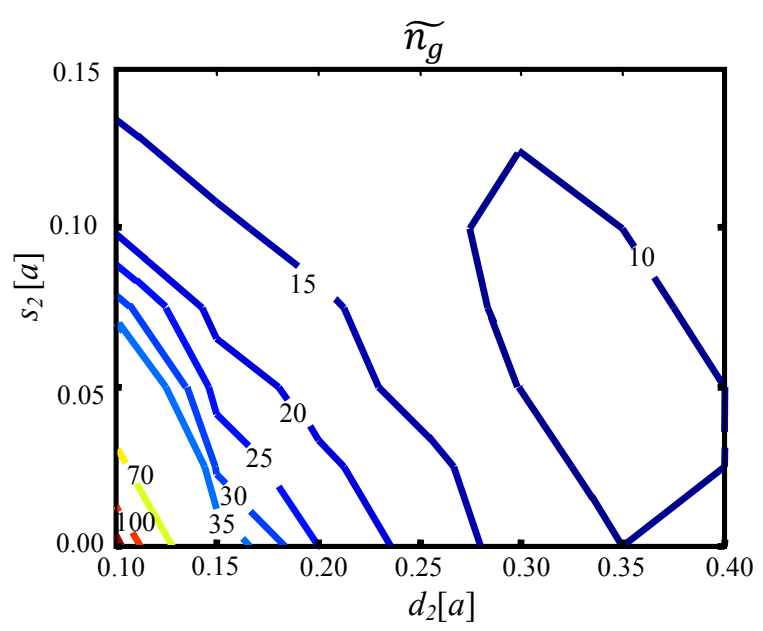

(b)

FIG. 6 (a) GBP and (b) average group index versus $d_{2}$ and $s_{2}$ for $d_{1}=0.3 a$ and $s_{1}=0.1 a$.

\begin{tabular}{|c|c|c|c|c|c|c|c|c|c|c|}
\hline Best structures & $\boldsymbol{d}_{1}$ & $\boldsymbol{s}_{1}$ & $\boldsymbol{d}_{2}$ & $\boldsymbol{s}_{2}$ & \multicolumn{3}{|c|}{ 2D Simulation } & \multicolumn{3}{|c|}{ 3D Simulation } \\
\cline { 6 - 11 } & {$[\mathrm{a}]$} & {$[\mathrm{a}]$} & {$[\mathrm{a}]$} & {$[\mathrm{a}]$} & $\mathrm{GBP}$ & $\tilde{n}_{g}$ & $\mathrm{BW}$ & GBP & $\tilde{n}_{g}$ & BW \\
\hline $\mathrm{A}$ & 0.3 & 0.1 & 0.15 & 0.1 & 0.332 & 16.0 & $32.2 \mathrm{~nm}$ & 0.348 & 21.0 & $24.5 \mathrm{~nm}$ \\
$\mathrm{~B}$ & 0.3 & 0.1 & 0.2 & 0.05 & 0.346 & 17.5 & $29.6 \mathrm{~nm}$ & 0.366 & 25.0 & $25.0 \mathrm{~nm}$ \\
$\mathrm{C}$ & 0.3 & 0.1 & 0.2 & 0.075 & 0.353 & 15.0 & $34.2 \mathrm{~nm}$ & 0.342 & 21.5 & $24.6 \mathrm{~nm}$ \\
$\mathrm{D}$ & 0.3 & 0.1 & 0.25 & 0.025 & 0.332 & 17.0 & $30.3 \mathrm{~nm}$ & 0.287 & 21.5 & $20.2 \mathrm{~nm}$ \\
\hline
\end{tabular}

TABLE 1 Best structures obtained in this paper and the corresponding $2 \mathrm{D}$ and $3 \mathrm{D}$ simulation results.

are $\mathrm{GBP}=0.247$ and $\tilde{n}_{g}=32$. We use this case in our next step of improvement.

Parallel shift of second row results in a descending shape dispersion curve and eliminates the minimum point as shown in Figure 5(a). To have single mode flat band regions we should analyze the effect of second row for $0.1 a \leq d_{2} \leq 0.4 a$. The simulation results show that shifting the second row in perpendicular direction $(y)$ does not improve GBP for negative values of $s_{2}$, and only positive values of $s_{2}$ between 0 and $0.15 a$ should be considered.

Figure 5(b) shows the dispersion curves for different values of $s_{2}$. The calculation results show that by increasing the value of $s_{2}$, the average group index increases and the bandwidth decreases. In these cases, the guided modes have descending shape and the central frequencies $\left(\omega_{0}\right)$ of guided modes shift to lower frequencies compared with waveguides obtained by only shifting the first row and thus the normalized bandwidth $\Delta \omega / \omega_{0}$ increases. Figures 6(a) and 6(b) show the GBP and average group index for different values of $d_{2}$ and $s_{2}$, respectively.

Using 2D simulation, we obtain four structures with GBP $>0.33$ which are listed in Table 1 . A small change in the shape of dispersion curve can cause a dramatic change in the corresponding GBP values. Since we have used effective refractive index in $2 \mathrm{D}$ simulation, we should validate the $2 \mathrm{D}$ simulation results by $3 \mathrm{D}$ simulations. The corresponding results of 3D simulations are provided in the same table. While the best structure in 2D simulation (C) has a GBP of 0.353 for $n_{g}=15$, we obtained a GBP of 0.366 and $n_{g}=25$ by 3D 


\begin{tabular}{|c|c|c|c|c|c|c|}
\hline & Ref. 9 & Ref. 10 & Ref. 11 & Ref. 13 & Ref. 14 & This paper \\
\hline Average group index $\left(\tilde{n}_{g}\right)$ & 34 & 11 & 32.5 & 37 & 90.177 & 25 \\
Bandwidth around 1550 nm & $11 \mathrm{~nm}$ & $43.45 \mathrm{~nm}$ & $14 \mathrm{~nm}$ & $8 \mathrm{~nm}$ & $6.37 \mathrm{~nm}$ & $25 \mathrm{~nm}$ \\
GBP & 0.22 & 0.31 & 0.293 & 0.19 & 0.35 & 0.366 \\
\hline
\end{tabular}

TABLE 2 Comparison of our engineered PCW with corresponding waveguides in literature.

simulation for the B structure. Figures 7(a) and 7(b) show the dispersion and group index curves, respectively for structures A, B, C and D computed by 3D PWE method. Figure 7(c) also depicts the dispersion parameter $D_{\lambda}$ corresponds to these four structures for the operating wavelength of $1550 \mathrm{~nm}$. As can be seen in this figure, $D_{\lambda}$ is less than $3 \mathrm{ps} / \mathrm{nm} \cdot \mathrm{mm}$ in slow light bandwidth for all proposed structures. Regarding to the GBP values achieved in 3D simulations, we consider waveguide B as "engineered waveguide" in the next section and design a structure to couple the light signal to the slow mode in this PCW.

Table 2 shows the results reported in literature on low dispersion slow light PCWs. In comparison to other papers, we have obtained higher group index-bandwidth product and a bandwidth of $25 \mathrm{~nm}$ which is more than the bandwidth required for DWDM systems. The structure proposed in Ref. [14] has the highest group index but in a narrow bandwidth. Also, the proposed structure in Ref. [10] has the largest bandwidth, but its group index is limited to 11 .

To complete the research, we analyze the sensitivity of GBP to variations in positions and radii of air holes caused by any fabrication the imperfections in the engineered PCW. We consider the imperfections in adjacent air holes and suppose deviation in position along $x$ and $y$ directions of first and second rows. These deviations are represented by $\Delta d_{1}, \Delta d_{2}, \Delta s_{1}$, and $\Delta s_{2}$ in Figure 8. Also, we define $\Delta r$ as a deviation in holes radii of the lattice.

Previous studies have shown that the deviation in position of air holes is less than $1 \mathrm{~nm}$ and in holes diameter is about $2 \mathrm{~nm}[26,27]$. Considering $a=380 \mathrm{~nm}$, we suppose maximum deviation of $\pm 0.004 a$ in position of air holes and $\pm 0.01 r$ in radii of air holes. In Figure $8, \triangle \mathrm{GBP}$ shows the error in value of GBP computed by 2D PWE analysis. The value of $\triangle \mathrm{GBP}$ is less than $5 \cdot 10^{-3}$ and $3 \cdot 10^{-2}$ for feasible technological deviations in position and radii of air holes, respectively.

There are some cases, such as $\Delta d_{1}=-0.004 a$ in Figure 8(a), in which $\triangle \mathrm{GBP}$ is a positive number. This indicates an improvement, although very small, in GBP. We should mention we have not claimed that the engineered structure is optimal, since the optimization required an extensive search in a $4 \mathrm{D}$ space $\left(d_{1}, d_{2}, s_{1}\right.$ and $\left.s_{2}\right)$.

\section{DESIGN OF COUPLER STRUCTURE FOR ENGINEERED PCW}

In the previous section, we obtained an "engineered waveguide" with high GBP and group index. In this section, we investigate the coupling to this waveguide. Among the methods

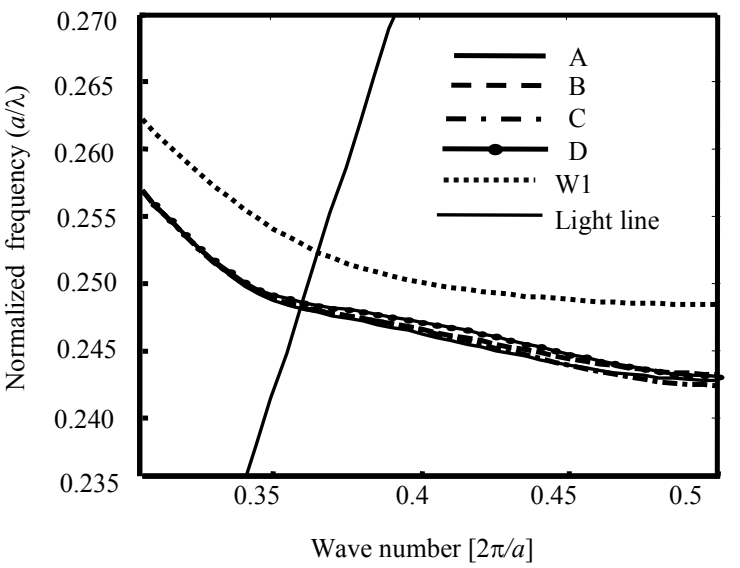

(a)

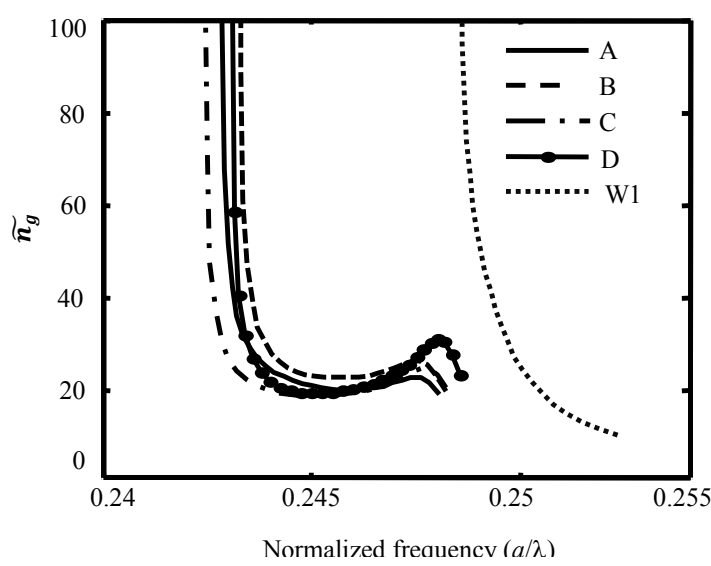

(b)

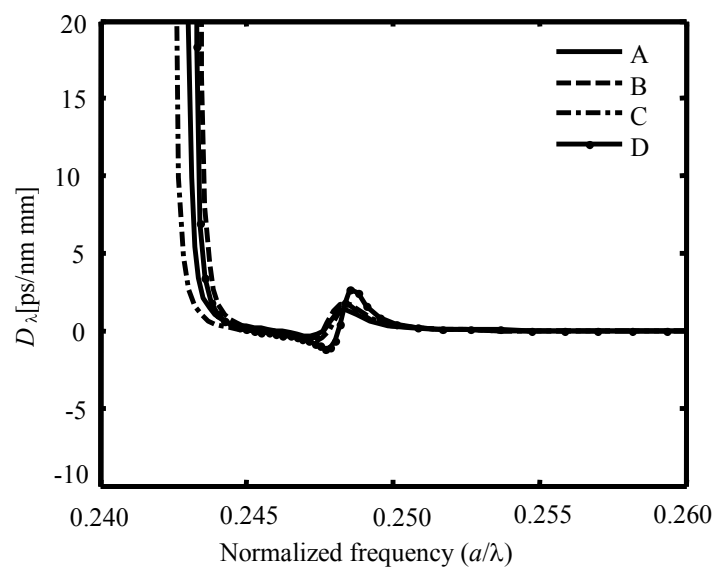

(c)

FIG. 7 (a) Dispersion curve, (b) group index versus frequency and (c) dispersion parameter $D$ for structures A, B, C and D defined in Table 1.

used to improve the coupling efficiency to $\mathrm{W} 1$ waveguide in the band edge of guided mode [16]-[20], using low group index section provides higher coupling efficiencies than PC tapers [23].

PCWs analyzed in previous research are mostly considered 
in the band edge and suffer from strong GVD. In this paper, we design a coupling waveguide which supports low groupindex mode for coupling to engineered waveguide in slow light frequency region. A coupling structure with a length of about $8 a$ is shown in Figure 9. Light is coupled from the strip waveguide to the low group-index mode in the coupler and then to the engineered PCW which supports slow light. We calculate the transmission spectra in the engineered PCW with emphasis on the slow light bandwidth by 2D finite difference time domain (FDTD) method [28, 29]. In this simulation, the grid size is chosen $d x=a / 16$ to have sufficient accuracy and low computation cost. Since the profiles of slow modes are wider than profiles of fast modes and also overlap with air holes adjacent to the waveguide, the monitor width in output should be considered large enough (6a) to cover the whole distributions of slow modes. Also, the boundary condition at the spatial edges of the computational domain is a perfectly matched layer (PML) which is one of the most effective absorbing boundary conditions [28]. The PML has a width of $0.5 a$ and a reflection of $10^{-8}$.

We consider two couplers. The first one, herein we call it "modified coupler", is a coupler with the same parameters
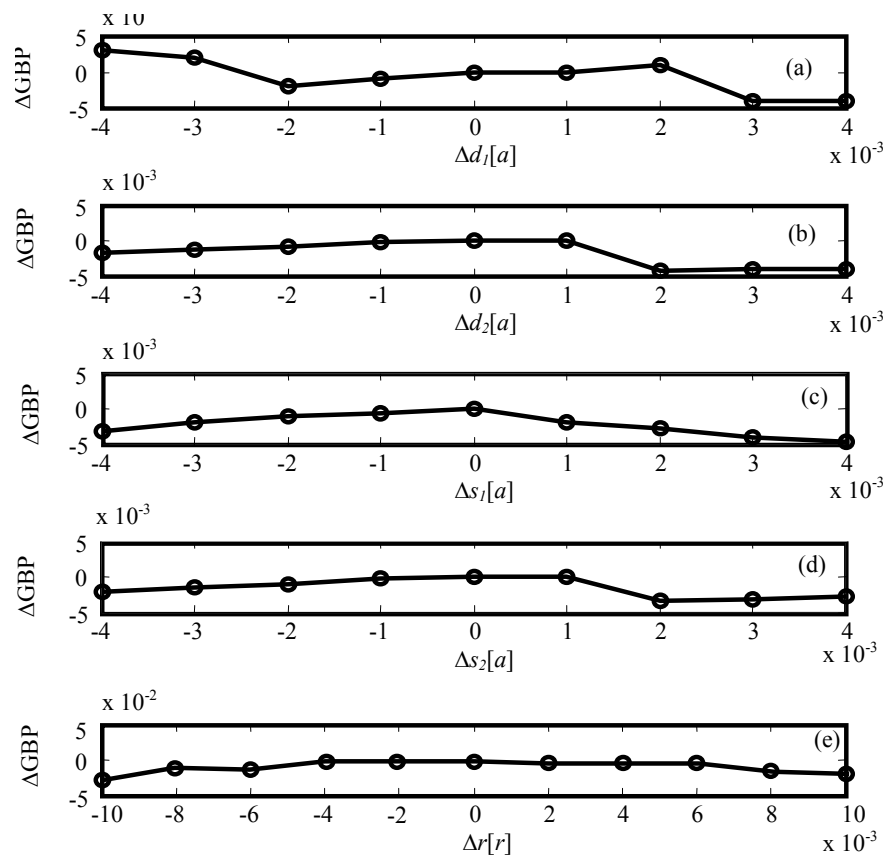

FIG. $8 \triangle \mathrm{GBP}$ versus deviations in position of air holes: (a) first row along $x$, (b) second row along $x,(c)$ first row along $y$ and (d) second row along $y$. (e) $\triangle G B P$ versus deviations in radii of air holes.

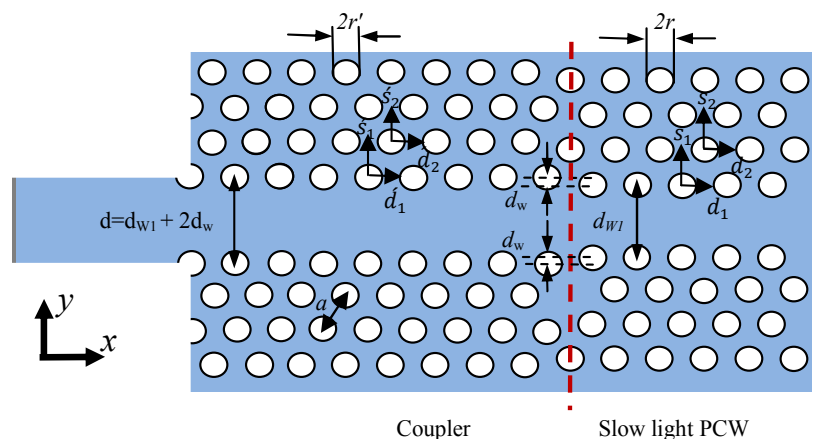

FIG. 9 Coupling structure to slow light waveguide and design parameters. as the engineered waveguide $\left(\hat{d}_{1}=0.3 a, \hat{s}_{1}=0.1 a, \hat{d}_{2}=0.2 a\right.$, $s_{2}=0.05 a$ ). The parameters with prime correspond to the coupler section, as shown in Figure 9. The second one, herein we call it "non-modified coupler", has the same parameters as the W1 waveguide $\left(\hat{d}_{1}=\hat{s}_{1}=\hat{d}_{2}=\hat{s}_{2}=0\right)$.

First, we consider the modified coupler. As shown in Figure 10(a), reducing the air holes radius of this modified coupler results in shifting the guided mode to lower frequencies. For example, for $r^{\prime}=0.25 a$ the fast and linear part of coupler mode overlaps with zero dispersion band of waveguide which is shown by hatched lines. Since changing the coupler width does not produce a mode with high group velocity in the flat band region, it is not an effective way to construct a low group index coupler. As shown in Figure 10(a), the coupler mode for $d w=0.1 a$ does not have high group velocity in the hatched region. Therefore, we only reduced the air holes radii and assumed a modified coupler with air holes radius of $r^{\prime}=0.25 a$ to evaluate the transmission spectrum.

Next, we consider the non-modified coupler. Guided modes of non-modified coupler, corresponding to $d w=0.1 a, d w=$ $0.15 a$ and $r^{\prime}=0.25 a$ are depicted in Figure 10(b). In these cases, the high group velocity part of the coupler appears in the slow light part of the engineered waveguide. Figure 11

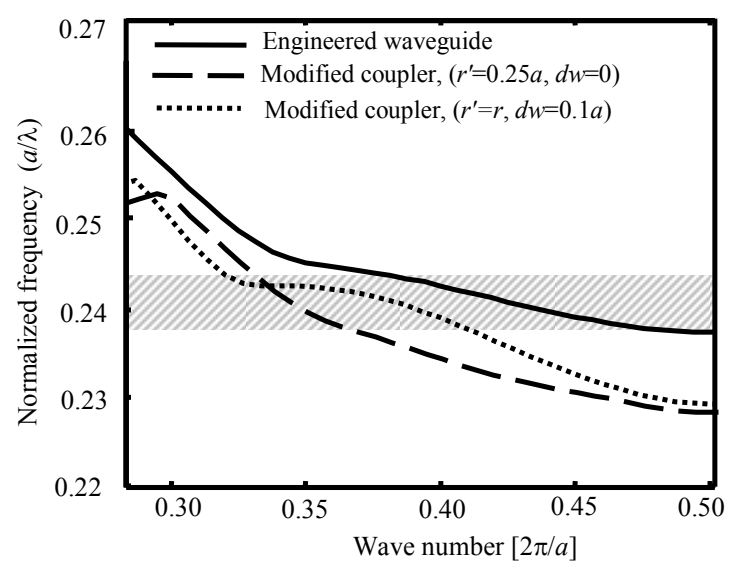

(a)

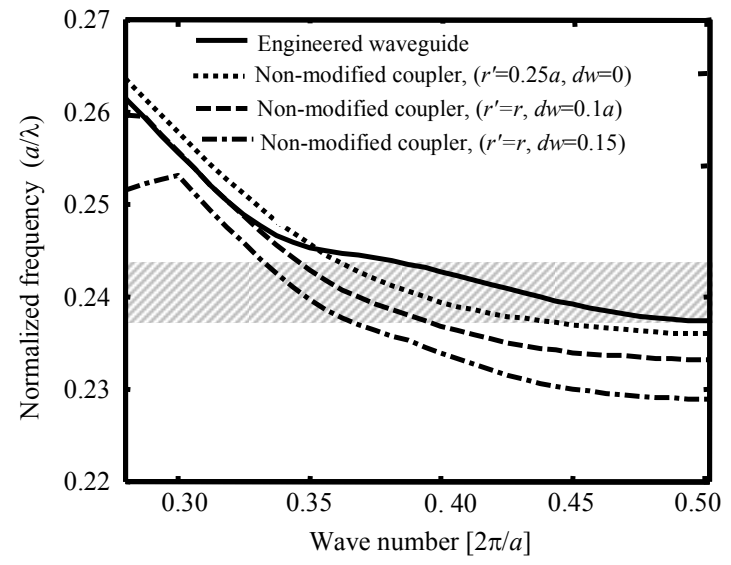

(b)

FIC. 10 (a) Dispersion curves of engineered waveguide and modified coupler ( $\dot{d}_{1}=0.3 a, \dot{s}_{1}=0.1 a, \dot{d}_{2}=0.2 a, \dot{s}_{2}=0.05 a$ ) for $r^{\prime}=0.25 a$ and $d w=0.1 a$ (b) Dispersion curves of engineerd waveguide and non-modified coupler $\left(\dot{d}_{1}=\dot{s}_{1}=\dot{d}_{2}=\dot{s}_{2}=0\right.$ ) for $r=0.25 a, d w=0.1 a$ and $d w=0.15 a$. 


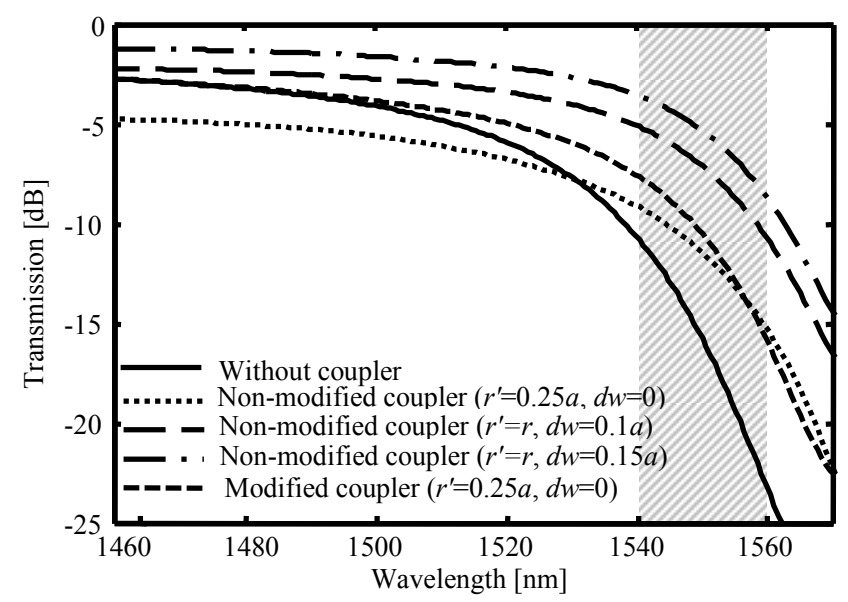

FIC. 11 The transmission spectra from strip waveguide to the engineered waveguide using modified and non-modified couplers and without coupler. Hatched region is zero dispersion slow light frequency band.

is depicted to evaluate the transmission spectra from strip waveguide to the engineered waveguide using modified and non-modified couplers and also without any coupler. The case of non-modified coupler and $d w=0.15 a$ produces the highest transmission in slow light bandwidth. It shows about $10 \mathrm{~dB}$ improvement at the wavelength of $1550 \mathrm{~nm}$ in the low dispersion region compared to the transmission without any coupler. The transmission to engineered waveguide without any coupler has $15 \mathrm{~dB}$ variation in low dispersion band. This variation reduces to $5 \mathrm{~dB}$ for the non-modified coupler $\left(r^{\prime}=r, d w=0.15 a\right)$ case. It means that the transmission is flatter in this case and causes less distortion in the signal pulse.

As discussed in previous papers [17]-[19], coupling efficiency in connection of strip waveguide to PCW also depends on the termination geometry of PCW. It is desired to improve transmission to low dispersion slow light in the engineered PCW. This is investigated by changing the parameter ti shown in Figure 12. We used $t_{i}=0$ in spectra shown in Figure 11. We can also cut the PCW at the boundaries of air holes $\left(t_{i}=0.286 a\right)$ and at the middle of the lattice period $\left(t_{i}=0.5 a\right)$. Both of these cases slightly improve the transmission efficiency (about $1 \mathrm{~dB}$ ) in the slow light frequency band. We suggest $t_{i}=0.5 a$ to be used in the proposed structure.

\section{CONCLUSIONS}

In this paper, we obtained low dispersion slow light in a photonic crystal waveguide by modifying the structure of lattice adjacent to the waveguide. Using this method, we achieved a GBP of 0.366 and an average group index of 25 in $25 \mathrm{~nm}$ bandwidth which is higher than the values reported in previous literatures. For utilization of the proposed PCW, we designed a coupling structure to obtain acceptable transmission efficiency to the slow light frequency band. Using this low group index coupler and adjusting the structure parameters, the transmission from the strip waveguide to the modified low-dispersion PCW was enhanced $11 \mathrm{~dB}$ in the zero dispersion region.

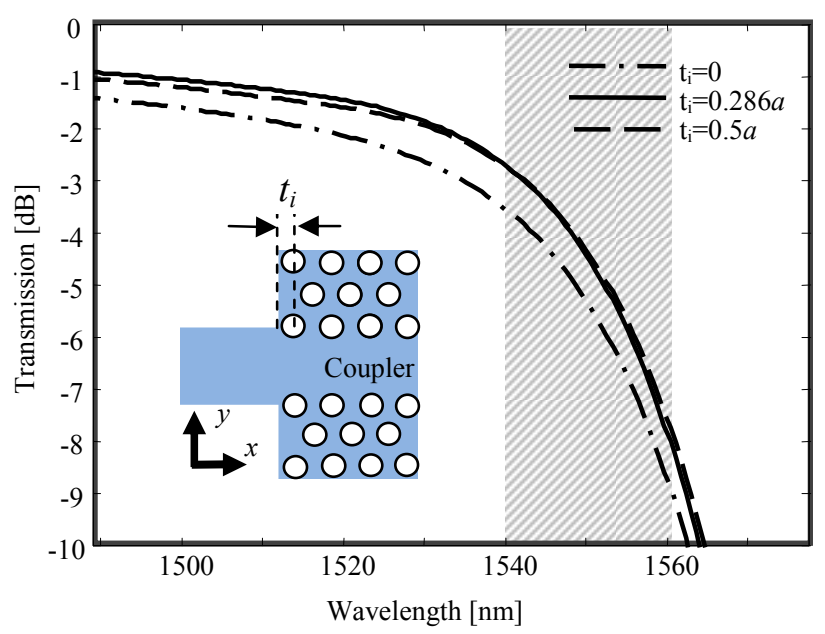

FIC. 12 Effect of final modification on transmission spectra from strip waveguide to the engineered waveguide using non-modified coupler $\left(r^{\prime}=r, d w=0.15 a\right)$ for different values of $t_{i}$.

\section{References}

[1] T. F. Krauss, "Why do we need slow light?," Nat. Photonics 2, 448-450 (2008).

[2] T. Baba, "Slow light in photonic crystals," Nat. Photonics 2, 465-473 (2008).

[3] T. F. Krauss, "Slow light in photonic crystal waveguides," J. Phys. D Appl. Phys. 40, 2666-2670 (2007).

[4] T. Baba, and D. Mori, "Slow light engineering in photonic crystals," J. Phys. D Appl. Phys. 40, 2659-2665 (2007).

[5] S. A. Schulz, L. O’Faolain, D. M. Beggs, T. P. White, A. Melloni, and T. F. Krauss, "Dispersion engineered slow light in photonic crystals: a comparison," J. Opt. 12, 104004 (2010).

[6] A. Yu. Petrov, and M. Eich, "Zero dispersion at small group velocities in photonic crystal waveguides," Appl. Phys. Lett. 85, 4866-4868 (2004).

[7] M. D. Settle, R. J. P. Engelen, M. Salib, A. Michaeli, L. Kuipers, and T. F. Krauss, "Flat band slow light in photonic crystals featuring spatial pulse compression and terahertz bandwidth," Opt. Express 15, 219-226 (2007).

[8] S. Kubo, D. Mori, and T. Baba, "Low-group-velocity and lowdispersion slow light in photonic crystal waveguides," 0 pt. Lett. 32, 2981-2983 (2007).

[9] L. H. Frandsen, A. V. Lavrinenko, J. F. Pedersen, and P. I. Borel, "Photonic crystal waveguides with semi-slow light and tailored dispersion properties," Opt. Express 14, 9444-9450 (2006).

[10] R. Hao, E. Cassan, H. Kurt, X. Le Roux, D. Marris-Morini, L. Vivien, $\mathrm{H}$. Wu, Z. Zhou, and X. Zhang, "Novel slow light waveguide with controllable delay-bandwidth product and utra-low dispersion," Opt. Express 18, 5942-5950 (2010).

[11] J. Li, T. P. White, L. O'Faolain, A. Gomez-Iglesias, and T. F. Krauss, "Systematic design of flat band slow light in photonic crystal waveguides," Opt. Express 16, 6227-6232 (2008).

[12] Y. Hamachi, S. Kubo, and T. Baba, "Slow light with low dispersion and nonlinear enhancement in a lattice-shifted photonic crystal waveguide," Opt. Lett. 34, 1072-1074 (2009).

[13] A. Säynätjoki, M. Mulot, J. Ahopelto, and H. Lipsanen, “Dispersion engineering of photonic crystal waveguides with ring-shaped holes," Opt. Express 15, 8323-8328 (2007). 
[14] R. Hao, E. Cassan, X. Le Roux, D. Gao, V. Do Khanh, L. Vivien, D. Marris-Morini, and X. Zhang, "Improvement of delay-bandwidth product in photonic crystal slow-light waveguides," Opt. Express 18, 16309-16319 (2010).

[15] L. O'Faolain, D. M. Beggs, T. P. White, T. Kampfrath, K. Kuipers, and T. F. Krauss, "Compact optical switches and modulators based on dispersion engineered photonic crystals," IEEE Photonics Journal 2, 404-414 (2010).

[16] A. Hosseini, X. Xu, H. Subbaraman, C. Lin, and R. T. Chen, "Large optical spectral range dispersion engineered silicon-based photonic crystal waveguide modulator," Opt. Express 20, 12318-12325 (2012).

[17] E. Miyai, and S. Noda, "Structural dependence of coupling between a two-dimensional photonic crystal waveguide and a wire waveguide," J. Opt. Soc. Am. B 21, 67-72 (2004).

[18] A. Mekis, and J. D. Joannopoulos, "Tapered couplers for efficient interfacing between dielectric and photonic crystal waveguides," J. Lightwave Technol. 19, 861-865 (2001).

[19] Y. A. Vlasov, and S. J. McNab, "Coupling into the slow light mode in slab-type photonic crystal waveguides," Opt. Lett. 31, 50-52 (2006).

[20] S. G. Johnson, P. Bienstman, M. A. Skorobogatiy, M. Ibanescu, E. Lidorikis, and J. D. Joannopoulos, "Adiabatic theorem and continuous coupled-mode theory for efficient taper transitions in photonic crystals," Phys. Rev. E 66, 1-15 (2002).

[21] P. Pottier, M. Gnan, and R. M. De La Rue, "Efficient coupling into slow-light photonic crystal channel guides using photonic crystal tapers," Opt. Express 15, 6569-6575 (2007).
[22] C.-Y. Lin, X. Wang, S. Chakravarty, B. S. Lee, W.-C. Lai, and R. T. Chen, "Wideband group velocity independent coupling into slow light silicon photonic crystal waveguide," Appl. Phys. Lett. 97, 183302 (2010).

[23] A. Hosseini, X. Xu, D. N. Kwong, H. Subbaraman, W. Jiang, and R. T. Chen, "On the role of evanescent modes and group index tapering in slow light photonic crystal waveguide coupling efficiency," Appl. Phys. Lett. 98, 031107 (2011).

[24] C. Martijn de Sterke, K. B. Dossou, T. P. White, L. C. Botten, and R. C. McPhedran, "Efficient coupling into slow light photonic crystal waveguide without transition region: role of evanescent modes," Opt. Express 17, 17338-17343 (2009).

[25] J. D. Joannopoulos, and S. G. Johnson, Photonic Crystals: The Road from Theory to Practice (Kluwer, Dordrecht, 2002).

[26] L. O'Faolain, T. P. White, D. O'Brien, X. Yuan, M. D. Settle and T. F. Krauss, "Dependence of extrinsic loss on group velocity in photonic crystal waveguides," Opt. Express 15, 13129-13138 (2007).

[27] D. M. Beggs, L. O'Foalain and T. F. Krauss, "Accurate determination of the functional hole size in photonic crystal slabs using optical methods," Photonic. Nanostruct. 6, 213-218 (2008).

[28] A. Taflove, and S. C. Hagness, Computational Electrodynamics: The Finite-Difference Time-Domain Method (third edition, Artech House Publishers, Norwood, 2005).

[29] A. Lavrinenko, P. I. Borel, L. H. Frandsen, M. Thorhauge, A. Harp $\phi$ th, M. Kristensen, T. Niemi, and H. M. H. Chong, "Comprehensive FDTD modeling of photonic crystal waveguide components," Opt. Express $12,234-248$ (2004). 